\title{
Novel wireless health monitor with acupuncture bio-potentials obtained by using a replaceable salt-water-wetted foam-rubber cushions on RFID-tag
}

\author{
Jium-Ming Lin ${ }^{\mathrm{a}, *}$, Hung-Han $\mathrm{Lu}^{\mathrm{b}}$ and Cheng-Hung Lin ${ }^{\mathrm{b}}$ \\ ${ }^{a}$ Department of Communication Engineering, Chung-Hua University, Hsin-Chu 30012, Taiwan \\ ${ }^{\mathrm{b}}$ Ph.D. Program in Engineering Science College of Engineering, Chung-Hua University, Hsin-Chu \\ 30012, Taiwan
}

\begin{abstract}
This paper proposes a bio-potential measurement apparatus including a wireless device for transmitting acupuncture bio-potential information to a remote control station for health conditions analysis and monitor. The key technology of this system is to make replaceable foam-rubber cushions, double-side conducting tapes, chip and antenna on the radio frequency identification (RFID) tag. The foam-rubber cushions can be wetted with salt-water and contact with the acupuncture points to reduce contact resistance. Besides, the double-side conducting tapes are applied to fix foam-rubber cushions. Thus, one can peel the used cushions or tapes away and supply new ones quickly. Since the tag is a flexible plastic substrate, it is easy to deploy on the skin. Besides, the amplifier made by CMOS technology on RFID chip could amplify the signals to improve $\mathrm{S} / \mathrm{N}$ ratio and impedance matching. Thus, cloud server can wirelessly monitor the health conditions. An example shows that the proposed system can be used as a wireless health condition monitor, the numerical method and the criteria are given to analyze eleven bio-potentials for the important acupunctures of eleven meridians on a person's hands and legs. Then a professional doctor can know the performance of an individual and the cross-linking effects of the organs.
\end{abstract}

Keywords: RFID tag, bio-potential, flexible substrate, wireless health monitor, wetted replaceable foam-rubber cushion

\section{Introduction}

Conventional biomedical probes made on a silicon substrate [1-7] are not only fragile but also unable to be engaged in the contour of the body, and the contact resistance between probe and skin may be increased. Besides, the signal processing devices are needed to improve $\mathrm{S} / \mathrm{N}$ ratio of the retrieved signals [8-11]. Dr. Voll discovered many electrically conductive points (acupuncture points) on the hands (and feet of the human body). The traditional bio-potential measuring devices can manually measure only one acupuncture point at a time, therefore, measuring the acupuncture points of a human subject to obtain stable and reliable skin bio-potential information is very time-consuming [12-21]. So

${ }^{*}$ Corresponding author: Jium-Ming Lin, Department of Communication Engineering, Chung-Hua University, Hsin-Chu 30012, Taiwan. Tel.: 886-3-5186483; Fax: 886-3-5186521; E-mail: jmlin@chu.edu.tw. 
this paper aims to provide a bio-potential measurement apparatus as shown in Figure 1(a), which includes a RFID-based reader and tags (Figure 1(b)) for transmitting acupuncture bio-potential information to a remote control station for analysis. Then, cloud server can wirelessly monitor the health conditions of persons. The key technology is to make a pair of foam-rubber cushions, double-side conducting tapes, RFID chip and antenna on an active RFID tag. The foam-rubber is a kind of soft material that can be wetted with salt-water and contact with the acupunctures on the skin to reduce the contact resistance. The two pieces of double-side conducting tapes are applied to fix a pair of salt-water wetted foam-rubber cushions on the RFID tag. Thus, one can peel the used foam-rubber cushions or double-side conducting tapes away, and supply a new one easily. Since the tag is a flexible substrate, e.g. PT, PET and PI, the modules are easier to deploy and conform to human body profile.

In this research, an assembly of a bio-potential measurement apparatus and a wireless device are made into a flexible band member wearable on the body, electrically coupled to a pair of foam-rubber cushions, and those devices can simultaneously measure the bio-potentials of acupuncture points. The RFID chip nearby can amplify the voltage signal by using a Complementary Metal-OxideSemiconductor Transistor (CMOS) technology with gain 10, and convert the signal into a digital one using an A/D converter. The measured bio-potential information can be transmitted to a remote control station (e.g. a RFID reader), which performs a Fourier transformation for analysis. Thus, the health conditions can be wirelessly monitored with a RFID reader [22-32] via the tag. An example is given to show that one can take those bio-potential measurements for a person at healthy conditions, and get the averaged values for normalization later. So, if there are new measurements later, one can obtain the normalized bio-potentials of the acupunctures. According to the distributions of the results, one can obtain the relative health conditions of the person under test. The major contribution of this paper is to make the measurement in an automatic manner, then one can accomplish the health condition analyses in quite a few minutes, and then a professional doctor can know the performance of individual organ and explain the cross-linking effects of the organs. The paper is organized as follows: the first section is an introduction; the next part illustrates the fabrication steps of probes and RFID antenna; Section 3 is simulation and discussion; the last part is a conclusion.

\section{Fabrication steps}

The steps to make the device on a flexible substrate are as follows:

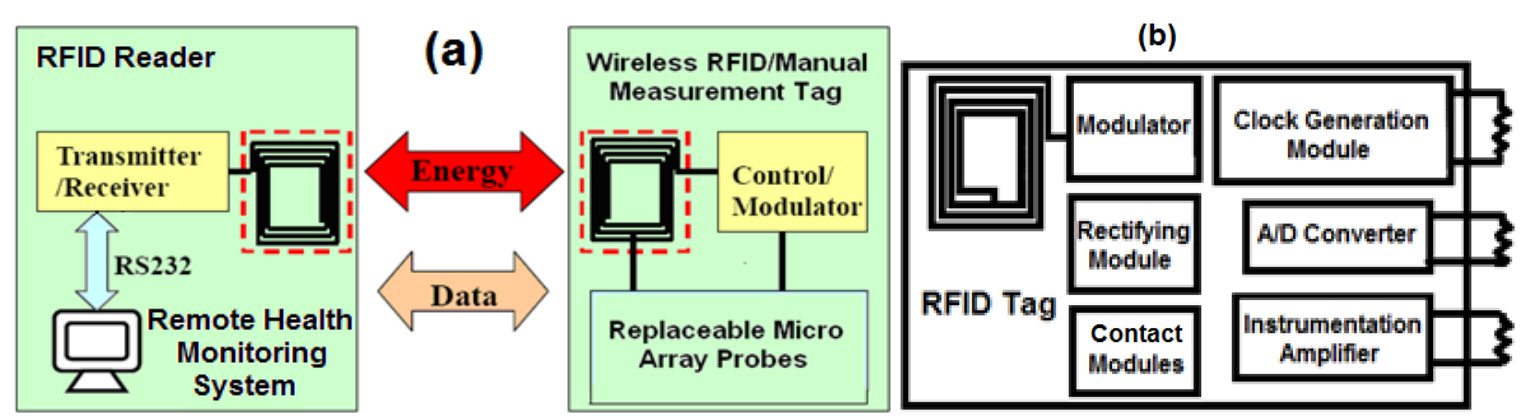

Fig. 1. The proposed RFID-based wireless health monitoring system: (a) System's setup; (b) Block diagram of the tag. 
Step 1: Deposit $\mathrm{SiO}_{2}$ on both surfaces of the substrate for thermal, electrical and humidity isolation. Cover the layer of positive Photo Resist (PR) on either surface to protect the layers of $\mathrm{SiO}_{2}$.

Step 2: Deposit a layer of $\mathrm{P}$ or N-type poly-silicon on the substrate, use mask 1 and Photolithography Processes (PAEP) to make the resistors. The resistors can be used for the circuits, such as RFID clock generation, A/D converter and amplifier in the RFID chip as shown in Figure $1 \mathrm{~b}$, to increase $\mathrm{S} / \mathrm{N}$.

Step 3: Use laser to go through holes on the substrate for conducting signals between foam-rubber cushion and RFID chip. Use mask 2 and PAEP to create a thick layer of SU-8 PR as Lift-Off-Resist (LOR). Evaporate layers of chrome and nickel to increase the adherence. Remove SU-8 PR, and plate a layer of gold. By the way, a battery socket is fixed on the tag so that the RFID monitor can operate on the active mode to extend the operating range; the result is shown in Figure 2. Screen printing silver paste as the contact bumps as shown in Figure 2 to adhere a double-side conducting tape later.

Step 4: A layer of a foam-rubber cushion is placed on a sheet of double-side conducting tape, and then cut into pieces and adhered on the contact bumps as shown in Figure 3. Coat the tag with an insulation layer, and only let the skin contact areas without insulating protection. It should be noted that the foam-rubber cushion should be soaked with salt water to reduce contact resistance. The flexible band can be wrapped in a ring shape and comprise a glove or a sock as shown in Figures 4 and 5, respectively.

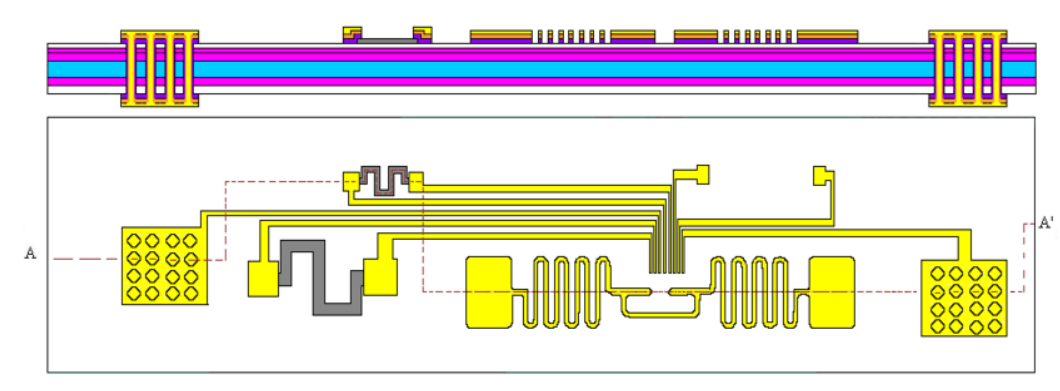

Fig. 2. Result of Step 3.

(b)

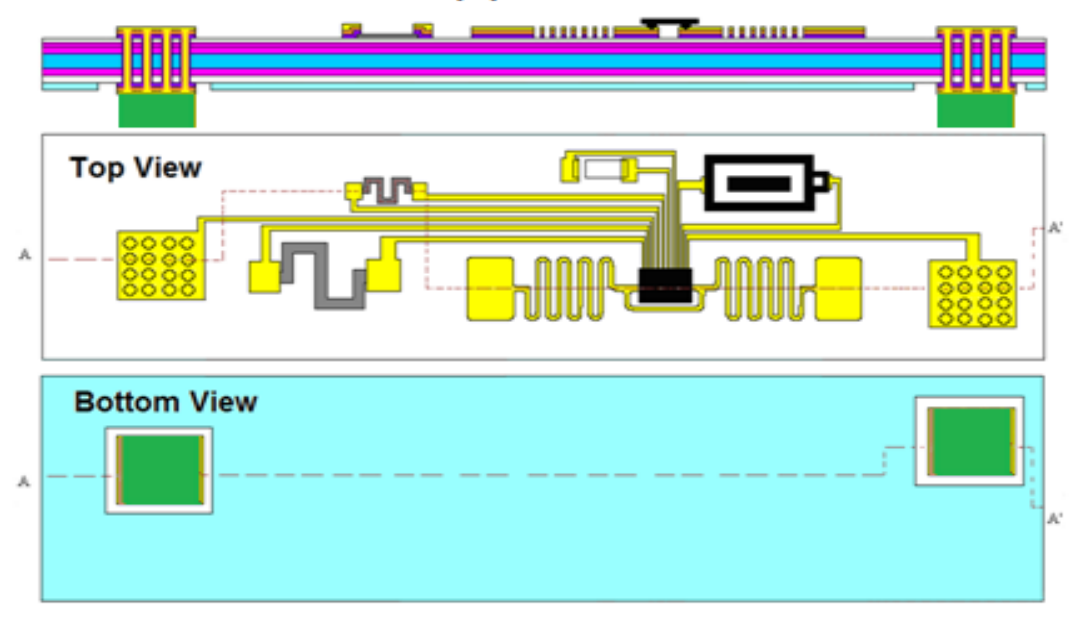

Fig. 3. The foam-rubber cushion is adhered on the bumps. 

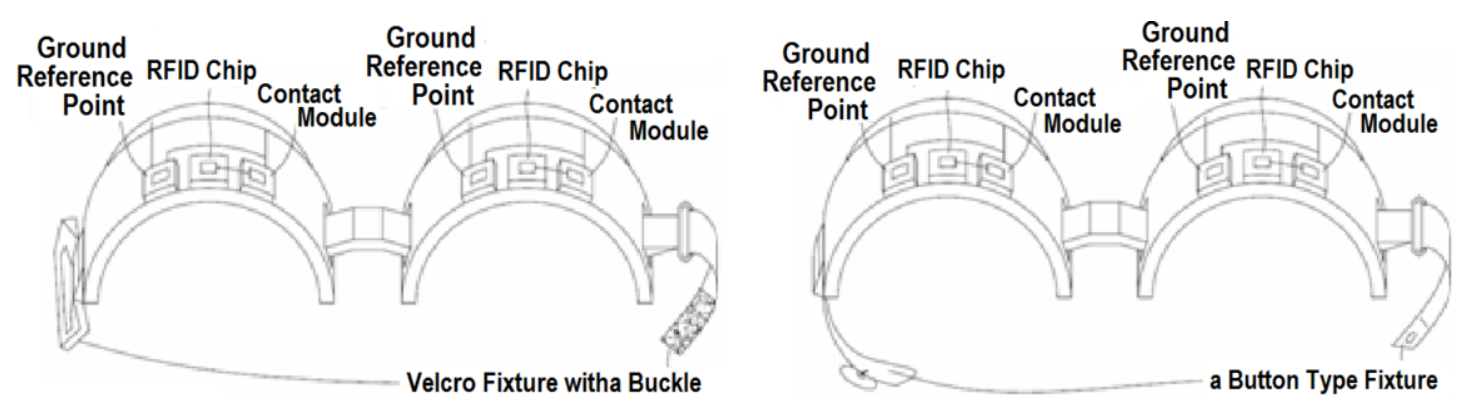

Fig. 4. The device can be covered over a finger with (a) a buckle on the Velcro fixture, and (b) a button type fixture.
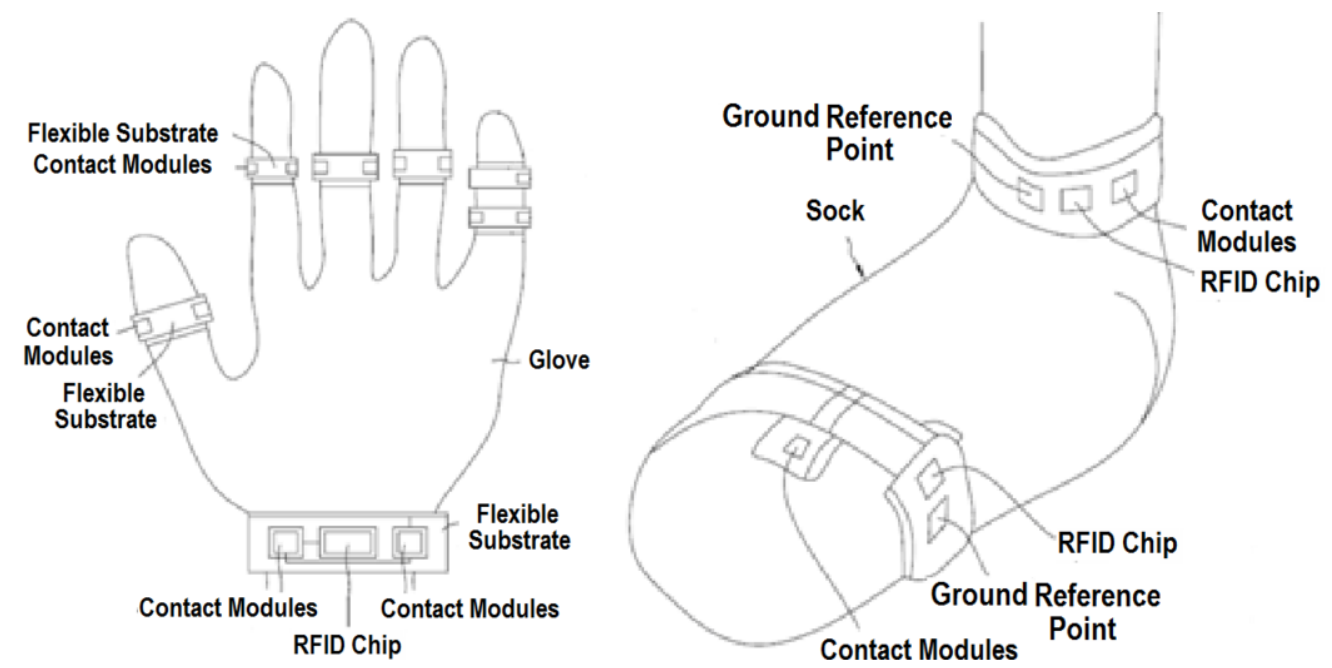

Fig. 5. The application by putting multiple tags on (a) fingers and wrist, and (b) the leg.

\section{System operation test and discussions}

The operation principle of the proposed device is as follows [29-32]. There are two modes of operation. The first one is to measure the acupuncture bio-impedance; the other is to measure the acupuncture bio-potentials. The processor on the RFID tag can decode the command from the RFID reader to take the analysis of some acupuncture points on a person. The circuit diagram and the circuit connections to integrate the RFID tag with the foam-rubber cushions are shown in Figures 6(a) and 6(b), respectively. In the first mode, the processor sends a pulse signal to the current source at point $\mathrm{A}$ as shown in Figure 6(b). Then the pulse current generator can output an impulse current signal to the acupuncture point, in which Q1-Q4 MOSFETs (Metal Oxide Semiconductor Field Effect Transistors) are in the RFID chip, where Q1 is a switch enabled by a pulse signal input voltage at point A, and Q2 is a current source by connecting gate to drain. The current output from point $\mathrm{C}$ is connected to the probe module adhered on the acupuncture under test. Then one can measure the impulse response voltage across the contact modules (across point $\mathrm{C}$ and the ground) as shown in Figure 6(b). To increase the $\mathrm{S} / \mathrm{N}$ ratio and improve the impedance matching problem, one can give the impulse response voltage to the instrumentation amplifier as shown in Figure 6(a), or connect to a CMOS amplifier with 

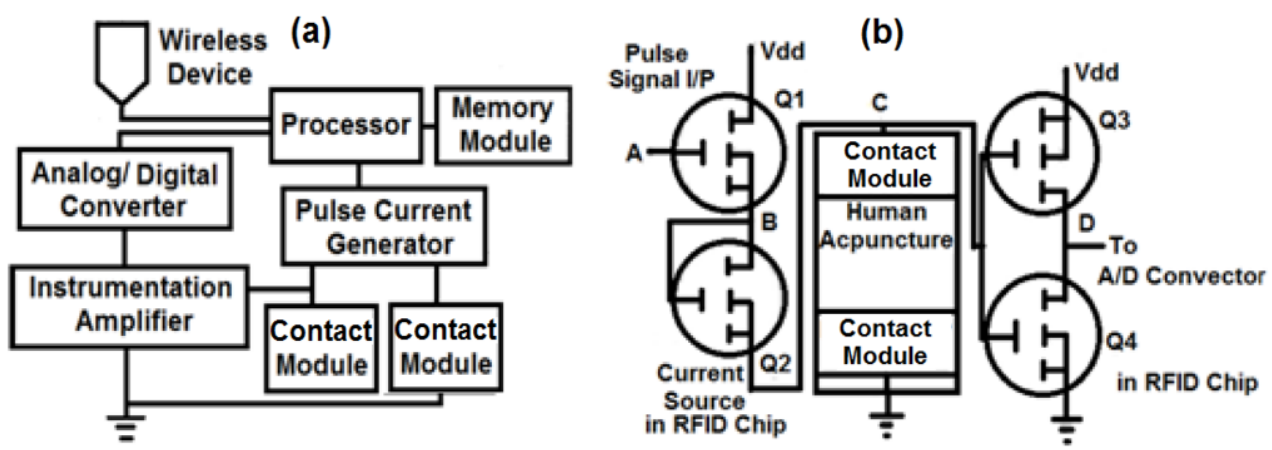

Fig. 6. (a) Circuit diagram to integrate the foam-rubber cushions with RFID Tag; (b) Detailed circuit connection.

gain 10 as shown in Figure 6(b) firstly, and then the amplified analog voltage at point $\mathrm{D}$ is converted to digital signal by an A/D converter in the active RFID chip.

Finally, the RFID tag can send the digital signal back to the RFID reader. After taking the FFT (Fast Fourier Transform) and signal conditioning processes, the RFID reader can deliver the acupuncture impedance to cloud server for further health condition analysis and monitor. This part is published in another paper [9] and will not be enclosed in this paper.

The second mode of operation is acupuncture bio-potential measurement. The circuit diagram to integrate the contact modules with RFID tag is the same as shown in Figures 6(a) and 6(b), but in this case, the processor does not send a pulse signal to the current source at point A. Instrumentation or a CMOS amplifier as shown in Figure 6(a) or 6(b) can amplify the acupuncture bio-potential signal across the contact modules of foam-rubber cushions (across point $\mathrm{C}$ and the ground) as shown in Figure 6(b). Then this bio-potential voltage at point $\mathrm{D}$ is converted into a digital signal by an A/D converter in the RFID chip. Finally, the RFID tag can send the digital signal back to the RFID reader for health condition analysis [33-36].

An example is given to measure eleven bio-potentials for the important acupunctures of eleven meridians on a person's hands and legs, such as taì bái (SP-3), shén mén (HE-7), yáng gŭ (SI-5), shù gǔ (BL-65), dà zhōng (KD-4), dà líng (PC-7), qiū xū (GB-40), taì chōng (LIV-3), tài yuān (LU-9), yáng $\mathrm{x} \overline{1}$ (LI-5), and chōng yang (ST-42). It is well known that the bio-potentials of these acupunctures are related to the meridian and human organ's functional performance as listed in Table 1.

Table 1

Relationships of acupunctures on hands and legs with human organ's function

\begin{tabular}{|l|l|l|}
\hline Meridian Number & Meridian Name & Acupuncture Name (Number) \\
\hline$* 1$ & Spleen-meridian & taì bái (SP-3) \\
\hline$* 2$ & Heart-meridian & shén mén (HE-7) \\
\hline$* 3$ & Small intestine-meridian & yáng gǔ (SI-5) \\
\hline$* 4$ & Bladder-meridian & shù gǔ (BL-65) \\
\hline$* 5$ & Kidney-meridian & dà zhōng (KD-4) \\
\hline$* 6$ & Pericardium-meridian & dà líng (PC-7) \\
\hline$* 7$ & Gallbladder-meridian & qiū xū (GB-40) \\
\hline$* 8$ & Liver-meridian & taì chōng (LIV-3) \\
\hline$* 9$ & Lung-meridian & tài yuān (LU-9) \\
\hline$* 10$ & Large intestine-meridian & yáng xī (LI-5) \\
\hline$* 11$ & Stomach-meridian & chōng yang(ST-42) \\
\hline
\end{tabular}




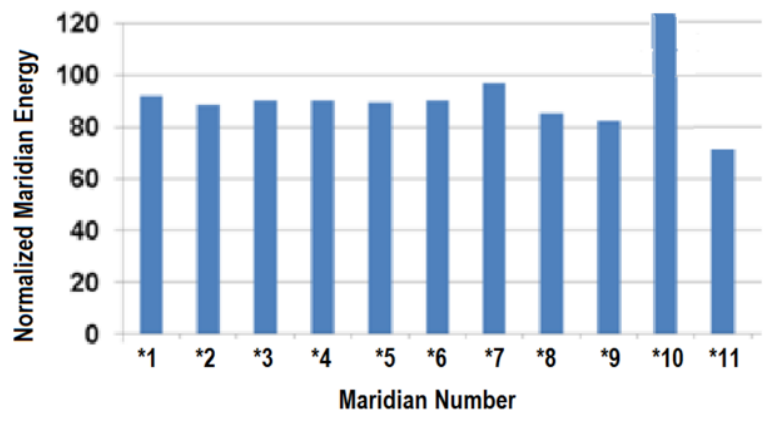

Fig. 7. Normalized energy distributions of a person's eleven meridians under test.

Since the electrical energy is proportional to the squared value of the voltage, the acupuncture impedance can be modeled as a parallel combination of an equivalent resistance and an equivalent capacitor. Meanwhile, it is quite familiar that the meridians are related to the nutrient and energy transportation path to the organs. Thus the squared value of a certain bio-potential is proportional to the nutrient and energy absorbed by the specified organ. So one can measure the bio-potentials for a person at healthy conditions, and find the averaged values of those nutrient and energy measurements for normalization later. Thereafter, if there are new measurements, one can obtain the normalized energy distributions of the meridians as shown in Figure 7. A larger value (e.g. above $+20 \%$ ) of a certain meridian energy (e.g. the large intestine-meridian as denoted by the tenth meridian in Figure 7) means that the organ is over-driven. On the contrary, if the value of a certain meridian energy is smaller (e.g. below $-20 \%$ ), the organ (e.g. the stomach-meridian as denoted by the eleventh meridian in Figure 7) will be driven by a lower meridian energy. So the proposed method and system can indicate whether some organs are in normal or in abnormal conditions. Then a qualified professional doctor can know the performance of individual organ and explain the cross-linking effects of the organs, and obtain a relative whole picture of the health conditions for the person under test.

\section{Conclusion}

This paper proposes a novel wireless health monitoring system. The key technology of this system is to make a replaceable non-invasive flexible foam-rubber cushions, RFID chip and antenna on an active RFID tag. The contribution of this paper is to make the acupuncture bio-potential measurement in a wireless and automatic manner, thus one can accomplish the health condition analyses in quite a few minutes, and then a professional doctor can know the performance of individual organ and explain the cross-linking effects of the organs.

\section{Acknowledgement}

National Science Council supported this research with the grants: NSC 101-2622-E-216-001-CC3, 101-2221-E-216-006-MY2, 101-2221-E-216-019-, and 102-2622-E-216-002-CC2. 


\section{References}

[1] M. Cormier, B. Johnson and M.K. Ameri, Fabrication and characterization of laser micromachined hollow microneedles, 12th International Conference on Transducers, Solid-State Sensors, Actuators and Microsystems 2 (2003), $1435-1438$.

[2] D. McAllisteria, F. Cros, S.P. Davis, L.M. Matta, M.R. Prausnitzu and M.G. Allen, Three-dimensional hollow microneedle and microtube arrays, J. of Micromechanics and Microengineering 14 (2004), 597-602.

[3] Z. Meng, M. Wang and M. Wong, High performance low temperature metal-induced unilaterally crystallized polycrystalline silicon thin film transistors for system-on-panel applications, IEEE Transactions on Electron Devices 47 (2000), 404-409.

[4] M. Wong, Z. Jin, G.A. Bhat, P.C. Wong and H.S. Kwok, Characterization of the MIC/MILC interface and its effects on the performance of MILC thin-film-transistors, IEEE Trans. on Electron Devices 47 (2000), 1061-1067.

[5] Jium-Ming Lin, Po-Kuang Chang and Zhong-Qing Hou, Bio-sensing and monitor system design with microarray probes and amplifier on an active RFID tag, Journal of Biomedical Engineering: Applications, Basis and Communications 21 (2009), 421-425.

[6] J. Rosell, J. Colominas, P. Riu, R. Pallas-Areny and J.G. Webster, Skin impedance from $1 \mathrm{~Hz}$ to $1 \mathrm{MHz}$, IEEE Trans. Bio-med. Eng. 35 (1988), 649-651.

[7] B. Brewitt, Methods for treating disorders by administering radio frequency signals corresponding to growth factors, U.S. Patent 5626617 (1997).

[8] O. Vahram, A. Valeri, M. Maksim, F. Gagik and H. Minas, Methods and devices for non-invasively measuring quantitative information of substances in living organisms, U.S. Patent 2005/0197555 (2005).

[9] L. E. Bertolucci, Nausea control device, U.S. Patent 4,981,146, Jan 1, 1991.

[10] T. L. Grey and L.E. Bertolucci, Electrotherapy device, U.S. Patent 5,397,338, Mar 14, 1995.

[11] D.M. Giuntoli, G.J. Gruzdowich and T L. Grey, Electro-acupuncture device with D-shaped stimulation electrodes, U.S. Patent No. 6,735,480, May 11, 2004.

[12] H.M. Johng, J.H. Cho, H.S. Shin, K.S. Soh, T.H. Koo and S.Y. Choi, Frequency dependence of impedances at the acupuncture point Queze (PC3), IEEE Engineering in Medicine and Biology 21 (2002), 33-36.

[13] A. Ahn, A.P. Colber, B.J. Anderson, O.G. Martinsen, R. Hammerschlag and S. Cina, Electrical properties of acupuncture points and meridians: A systematic review, Bioelectromagnetics 29 (2008), 245-256.

[14] X. Wang, B. Ma, Z. Gan and H. Zhang, Drop test and simulation of portable electronic devices, 6th International Conference on Electronic Packaging Technology, 2005, 1-4.

[15] B. Chen, J. Wei, E. Tay, Y.T. Wong and C. Iliescu, Silicon microneedle array with biodegradable tips for transdermal drug delivery, Microsyst. Technol. 14 (2008), 1015-1019.

[16] J.H. Park, M.G. Allen and M.R. Prausnitz, Biodegradable polymer microneedles: fabrication, mechanics and transdermal drug delivery, Journal of Controlled Release 104 (2005), 51-66.

[17] L. Lin and A. Pisano, Silicon-processed microneedles, Microelectromechanical Systems 8 (1999), $78-84$.

[18] J.D. Zahn, N.H. Talbot, D. Liepmann and A.P. Pisano, Microfabricated polysilicon microneedles for minimally invasive biomedical devices, Biomedical Microdevices 2 (2000), 295-303.

[19] M. Cormier, B. Johnson and M.K. Ameri, Fabrication and characterization of laser micromachined hollow microneedles, Transducers, Solid-State Sensors, Actuators and Microsystems 2 (2003), 1435-1438.

[20] K. Kontturi and L. Murtomäki, Impedance spectroscopy in human skin: a refined model, Pharmaceutical Research 11 (1994), 1355-1357.

[21] K.G. Chen, Electrical properties of meridians: with an overview of the electro-dermal screening test, IEEE Eng. Med./Biology Magazine 58 (1996), 58-63.

[22] H. Alemdar and C. Ersoy, Wireless sensor networks for healthcare: a survey, Computer Networks 54 (2010), 26882710.

[23] C.C. Lin, M.J. Chiu, C.C. Hsiao, R.G. Lee and Y.S. Tsai, Wireless health care service system for elderly with dementia, IEEE Trans. Inf. Technol. Biomed. 10 (2006), 696-704.

[24] A. Milenkovic, C. Otto and E. Jovanov, Wireless sensor networks for personal health monitoring: Issues and an implementation, Computer Communications 29 (2006), 2521-2523.

[25] E. Jovanov, A. Lords, D. Raskovic, P. Cox, R. Adhami and F. Andrasik, Stress monitoring using a distributed wireless intelligent sensor system, IEEE Engineering in Medicine and Biology Magazine 22 (2003), 45-49.

[26] J.P. Lynch and K.J. Loh, A summary review of wireless sensors and sensor, The Shock and Vibration Digest 38 (2006), 91-128.

[27] E. Sardini and M. Serpelloni, Instrumented wearable belt for wireless health monitoring, Procedia Engineering 5 (2010), $580-583$. 
[28] Y. Yamamoto, Measurement and analysis of skin electrical impedance, Acta. Dermal-Venereologist 185 (1994), $34-38$.

[29] G. López, V. Custodio and J. I. Moreno, LOBIN: E-textile and wireless-sensor-network-based platform for healthcare monitoring in future hospital environments, IEEE Transactions on Information Technology in Biomedicine 14 (2010), $1446-1458$.

[30] Y. Xiao, D. Takahashi, J. Liu, H. Deng and J. Zhang, Wireless telemedicine and m-health: technologies, applications and research issues, International Journal of Sensor Networks 10 (2011), 202-236.

[31] H. Huo, Y. Xu, H. Zhang, Y.H. Chuang and T.C. Wu, Wireless-sensor-networks-based healthcare system: a survey on the view of communication paradigms, International Journal of Ad Hoc and Ubiquitous Computing 8 (2011), 135-154.

[32] V. Chawla and D.S. Ha, An overview of passive RFID, IEEE Appl. Practice 45 (2007), 11-17.

[33] W. Yao, C.H. Chu and Zang Li, Leveraging complex event processing for smart hospitals using RFID, Journal of Medical Systems 34 (2011), 799-810.

[34] M. Safkhani, N. Bagheri and M. Naderi, On the designing of a tamper resistant prescription RFID access control system, Journal of Medical Systems 36 (2012), 3995-4004.

[35] E.W.T. Ngai, K.K.L. Moon, F.J. Riggins and C.Y. Yi, RFID research: An academic literature review (1995-2005) and future research directions, International Journal of Production Economics 112 (2008), 510-520.

[36] B.S. Ashar and A. Ferriter, Radio frequency Identification technology in health care benefits and potential risks, The Journal of the American Medical Association 298 (2007), 2305-2307. 